\title{
Circadian Control of cab Gene Transcription and mRNA Accumulation in Arabidopsis
}

\author{
Andrew J. Millar and Steve A. Kay' \\ Laboratory of Plant Molecular Biology, The Rockefeller University, 1230 York Avenue, New York, New York \\ $10021-6399$
}

An intriguing property of many organisms is their ability to exhibit rhythmic cellular events that continue independently of environmental stimuli. These rhythmic processes are generated by an endogenous mechanism known as the biological clock. We wished to determine whether Arabidopsis thaliana will serve as a model plant system for a molecular genetic dissection of the circadian clock. To this end, we investigated the expression of Arabidopsis chlorophyll $a / b$-binding protein (cab) genes throughout the circadian cycle. Steady-state mRNA levels of the cab2 and cab3 genes showed a dramatic circadian cycling in plants shifted from light/dark cycles to constant darkness, whereas the cab1 mRNA level exhibited little or no cycling under the same conditions. Analysis of cab promoter fusions in transgenic tobacco revealed that both the cab1 and cab2 $5^{\prime}$ upstream regions confer circadian-regulated expression on a chloramphenicol acetyltransferase (cat) reporter gene. In vitro nuclear run-on transcription assays also indicated that the transcription of the cab1 and cab2 genes is circadian regulated in Arabidopsis. Taken together, these data suggest that a post-transcriptional mechanism influences cab1 mRNA levels in Arabidopsis. The identification of circadian-regulated cis-acting elements in the cab1 and cab2 upstream regions will provide powerful tools for both molecular and genetic analysis of the higher plant circadian clock.

\section{INTRODUCTION}

Many important cellular processes are observed to occur rhythmically with peak activity appearing once every 24 $\mathrm{hr}$. These rhythms have been demonstrated to be under endogenous control, as they also persist under constant environmental conditions (Edmunds, 1988). The cellular machinery that generates circadian rhythms is known as the biological clock. Circadian rhythms have been observed in almost all eukaryotes appropriately examined (Rosbash and Hall, 1989) as well as in some prokaryotes (Huang et al., 1990). Interestingly, several properties of these rhythms are shared among these diverse species, including entrainment to natural day/night cycles by light and the ability to compensate for changes in ambient temperatures (Edmunds, 1988). An understanding of the molecular components that constitute these ubiquitous pacemakers will have, therefore, broad significance for many organisms. This is most pertinent where studies of circadian processes can be performed in organisms that are easily amenable to both genetic and molecular analysis, as has been shown in Drosophila (Rosbash and Hall, 1989; Young et al., 1989) and Neurospora (Dunlap, 1990).

Higher plants have played an important historical role in the study of biological clocks (Sweeney, 1987). Initially, the rhythmic "sleep" movements observed in leaves of

\footnotetext{
${ }^{1}$ To whom correspondence should be addressed.
}

some legumes provided an excellent system for study (Simon et al., 1976; Millet et al., 1989). In addition to the morphological rhythms, plants have revealed numerous circadian oscillations at the physiological and biochemical levels. Processes as diverse as stem elongation (Lecharny and Wagner, 1984; Lecharny et al., 1990), stomatal opening and closure (Gorton et al., 1989), and many specific enzyme activities (Deng et al., 1989) exhibit circadian control. In most instances, these rhythms have been shown to be reset by light, and in several cases the photoreceptor that mediates the resetting stimulus has been shown to be phytochrome (Simon et al., 1976). However, the interaction between phytochrome and the circadian clock currently remains unclear.

More recently, the levels of specific mRNAs have been shown to be under circadian control in animal (Uhl and Reppert, 1986), fungal (Loros and Dunlap, 1991), and plant systems (Kay and Millar, 1990). In the case of higher plants, several investigators have documented the diurnal and circadian fluctuation in the level of mRNAs encoding the light-harvesting chlorophyll $a / b$-binding proteins of photosystem II (reviewed in Kay and Millar, 1990). Although these observations have been made in diverse species under different experimental regimes, several major properties can be summarized concerning circadian-regulated $c a b$ gene expression: cab mRNA levels peak in the late 
morning and reach a minimum in the late evening; this rhythmic fluctuation persists in constant darkness with a periodicity of approximately $24 \mathrm{hr}$ but undergoes a significant damping in peak level; dramatic alteration of the light/ dark regime can alter the phase of the cycling (Giuliano et al., 1988), and the cycling of cab mRNAs is more dramatic in some species than others (Meyer et al., 1989). The circadian clock has been shown to regulate cab gene expression at the transcriptional level, initially by study of the wheat cab1 gene in transgenic tobacco (Nagy et al., 1988a) and later by in vitro nuclear run-on assays in several plant species (Giuliano et al., 1988; Paulsen and Bogorad, 1988; Taylor, 1989). These studies demonstrated that the clock acted directly upon transcription, and this also has been shown recently for the Neurospora ccg genes (Loros and Dunlap, 1991). In the case of the wheat cab1 gene, a 268-bp promoter fragment has been shown to mediate clock-regulated, as well as phytochrome-regulated, transcription in transgenic tobacco (Nagy et al., 1988a; Fejes et al., 1990).

Although the identification of a cis-acting DNA element for circadian regulation is a major step forward in the molecular analysis of the biological clock, several problems hinder rapid progress in the plant field. First, with a few exceptions (Nagy et al., 1988a; Stayton et al., 1989), most investigators have analyzed the sum of the cab gene family transcription and, therefore, may have missed the possibility of differential regulation among individual family members. Second, the studies have not been performed in a plant species that can also offer a straightforward genetic approach to understanding the components of the clock. Therefore, we have initiated studies on circadian-regulated gene expression in Arabidopsis to determine whether this organism can be used as a model system for a molecular genetic analysis of the higher plant circadian clock. Besides its well-documented advantages for genetic analysis (Redei, 1970; Estelle and Somerville, 1986), Arabidopsis has a small and well-characterized cab gene family consisting of three highly homologous members (Leutweiler et al., 1986; Karlin-Neumann et al., 1988; Mitra et al., 1989) and a more divergent homolog that has been detected in DNA gel blots (Leutweiler et al., 1986).

The previous characterization of this gene family has allowed us to study the effect of the clock on specific cab genes. Surprisingly, one of these genes, cab1, did not exhibit an mRNA fluctuation, whereas the other two, cab2 and cab3, showed a dramatic circadian cycling. Analysis of translational and transcriptional fusions of the cab promoters with a chloramphenicol acetyltransferase (cat) reporter gene in transgenic tobacco demonstrated that all three promoters are similarly regulated by the circadian clock. Consistent with these data, we also showed by using in vitro nuclear run-on transcription assays that the transcription rate of both the cab1 and cab2 genes is circadian regulated in Arabidopsis. We hypothesize, therefore, that post-transcriptional events result in a stabilization of the cab1 mRNA in Arabidopsis and conclude that this organism will provide a powerful system for both genetic and molecular dissection of the biological clock.

\section{RESULTS}

\section{The Level of mRNA Encoding the Arabidopsis cab Gene Family Exhibits a Low Amplitude Circadian Cycling}

It has been shown previously that some plant species exhibit a very poor cycling of cab mRNA throughout the circadian cycle (Meyer et al., 1989), preventing a study of the clock with this marker. Therefore, we investigated the cycling of cab mRNA in Arabidopsis to test whether the $c a b$ genes would be useful markers for studying circadian regulation in this species. Plants were grown from seed for 3 weeks in a growth chamber under a 12-hr light/12$\mathrm{hr}$ dark (12L12D) regime. We began harvesting plants at the onset of illumination, and after $12 \mathrm{hr}$ of illumination, the plants were placed in constant darkness (DD) for the following $50 \mathrm{hr}$. Samples were taken every $2 \mathrm{hr}$ and frozen, and total RNA was prepared from each sample and analyzed for cab gene family mRNA in slot blots using a coding sequence probe. Figure 1 shows the results of this analysis. The sampling time was expressed in hours as zeitgeber time (ZT) (Zerr et al., 1990), which is simply the number of hours after the onset of illumination. Zeitgeber

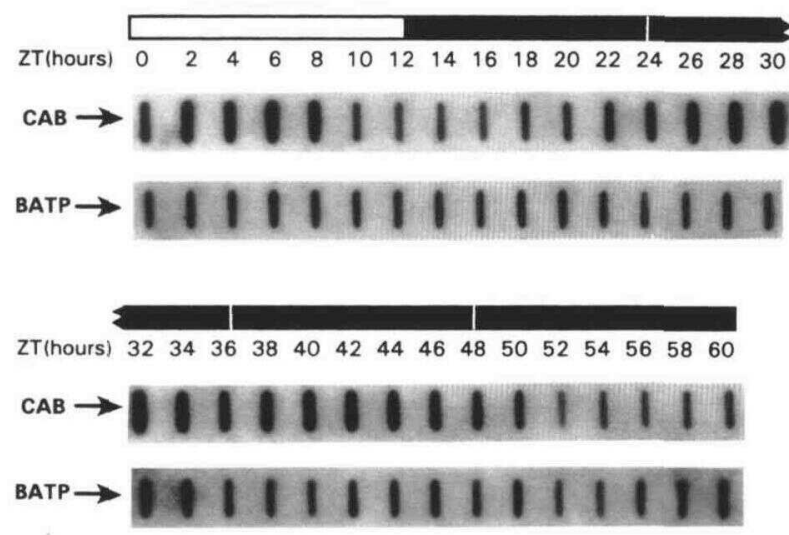

Figure 1. RNA Slot Blot Analysis of the cab Gene Family in Arabidopsis.

Total RNA was prepared from plants harvested every $2 \mathrm{hr}$ during one LD cycle followed by DD. A slot blot of these samples was hybridized to coding region probes from Arabidopsis cab1 (CAB) and the $\beta$-subunit of the mitochondrial ATPase (BATP). The open box above the time points marks the light period; the filled box indicates the period of constant darkness. Ten micrograms of RNA was loaded in each slot. ZT (hours), zeitgeber time (in hours). 
is a common term used in chronobiology for the environmental signal(s) that resets the circadian clock. ZT is used to allow comparison of experiments conducted under different light/dark (LD) regimes (Zerr et al., 1990).

The cab mRNA level clearly fluctuated throughout the first cycle (ZTO to ZT24), with a peak at ZT6 in the first day and another broad peak at ZT30 in DD (Figure 1). This periodicity of approximately $24 \mathrm{hr}$ was similar to that observed for cab mRNA levels in several other plant species (Kay and Millar, 1990). In contrast to the cab mRNA level, no significant cycling in the level of the mRNA encoding the $\beta$-subunit of the mitochondrial ATPase could be detected (Figure 1). The amplitude of the cab mRNA level from peak (ZT6) to trough (ZT14) as determined by densitometry was only threefold, which is significantly lower than the 20-fold to 50-fold amplitude observed in other species such as wheat, tobacco, and tomato (Giuliano et al., 1988; Nagy et al., 1988a; Paulsen and Bogorad, 1988). These data demonstrated that the Arabidopsis cab gene family mRNA level is regulated by an endogenous circadian clock, albeit at a lower amplitude than some other species. The peak in cab mRNA level in DD was very broad, and only a shallow dip in expression level could be detected from ZT36 to ZT40. From ZT46 onward, the cab gene family mRNA level decreased to a basal level of $45 \%$ of the maximal, indicating a rapid damping of the cab mRNA rhythm in DD. In several other species, at least two peaks in cab mRNA level have been observed in DD (Kay and Millar, 1990).

\section{Circadian Regulation of Arabidopsis cab mRNA Levels Is Gene Specific}

From these results, it would appear that the cab mRNA level only cycles weakly in Arabidopsis and is not, therefore, a suitable marker for studying the circadian clock in this plant. However, the Arabidopsis cab gene family is composed of three major members: cab1, cab2, and cab3 (Leutweiler et al., 1986; Mitra et al., 1989). All three genes are linked tightly, with cab1 being transcribed in the opposite direction to the tandemly arranged cab2 and cab3 (Leutweiler et al., 1986). The coding regions of these three genes are conserved very highly at the nucleic acid level $(96 \%)$, and the high homology between cab2 and cab3 extends into the $3^{\prime}$ and $5^{\prime}$ untranslated regions, as well as to approximately 190 bp upstream of the transcription start site (Leutweiler et al., 1986; Mitra et al., 1989). cab1 has diverged from cab2/cab3 in the noncoding regions of the gene. Because cab2 and cab3 are so highly homologous, it is technically difficult to distinguish between their respective mRNAs on a routine basis. Therefore, we prepared a single-stranded DNA probe that can distinguish between cab1 and the cab2/cab3 mRNAs in S1 nuclease protection experiments. This enabled us to investigate the circadian regulation of the cab mRNAs on a gene-specific basis.

Figure 2 shows the results of an $\mathrm{S} 1$ nuclease protection analysis using the same RNA samples described in Figure 1. The signal derived from protection of the probe by cab2/ cab3 mRNAs shows a dramatic oscillation throughout the experimental period. Starting from ZTO, the cab2/cab3 mRNA level peaked around ZT4 and declined to very low levels at ZT14, and a strong peak returned in DD around ZT28, indicating a periodicity of about $24 \mathrm{hr}$. An additional band is present between the $c a b 2 / 3$ and $c a b 1$ signals that is a breakdown product of the cab2/3 band. The cab2/ cab3 mRNA levels varied by approximately 25 -fold between maximum and minimum, and after ZT36 the signal declined to a very low level at ZT52. In striking contrast to the cab2/cab3 mRNAs, the cab1 mRNA remained relatively constant throughout the entire experimental period, with no more than a twofold variation and a small decline observed at ZT54.

Taken together, these results demonstrated that the cab2 and cab3 mRNA levels are strongly regulated in a circadian fashion, whereas the cab1 mRNA level is only weakly, if at all, regulated by the circadian clock. The summation of these signals at each time point resulted in the low amplitude fluctuation observed in the slot blot analysis shown in Figure 1. Although minor differences in the cycling of specific cab mRNAs had been reported previously in petunia (Stayton et al., 1989), all of the steady-state $c a b$ mRNA levels exhibited clear cycling, unlike our observations for the cab1 gene in Arabidopsis.

\section{Transcription of Both the cab1 and cab2 Genes Is Regulated by the Circadian Clock}

The differential expression of the Arabidopsis cab genes during the circadian cycle could result from differences in either transcription, mRNA turnover, or both during the circadian cycle. To investigate which of these possibilities were operating, we examined the expression of both translational and transcriptional cab-cat fusions in transgenic tobacco. Seeds of the translational fusions were kindly provided by Dr. Gynheung An (Washington State University), who has demonstrated that these fusions are correctly expressed in transgenic tobacco in terms of light/ dark and organ-specific regulation (An, 1987). The transgenic tobacco seeds were germinated and grown for 2 weeks under a $16 \mathrm{~L} 8 \mathrm{D}$ regime, after which the seedlings were entrained to $12 \mathrm{~L} 12 \mathrm{D}$ for $48 \mathrm{hr}$. Samples of seedlings were collected and frozen every $3 \mathrm{hr}$ from an initial LD cycle, followed by continuous darkness. RNA was isolated and the expression of the chimeric cab-cat mRNA was assayed in RNA gel blots using a radiolabeled probe derived from the cat gene. 
ZT(hours)
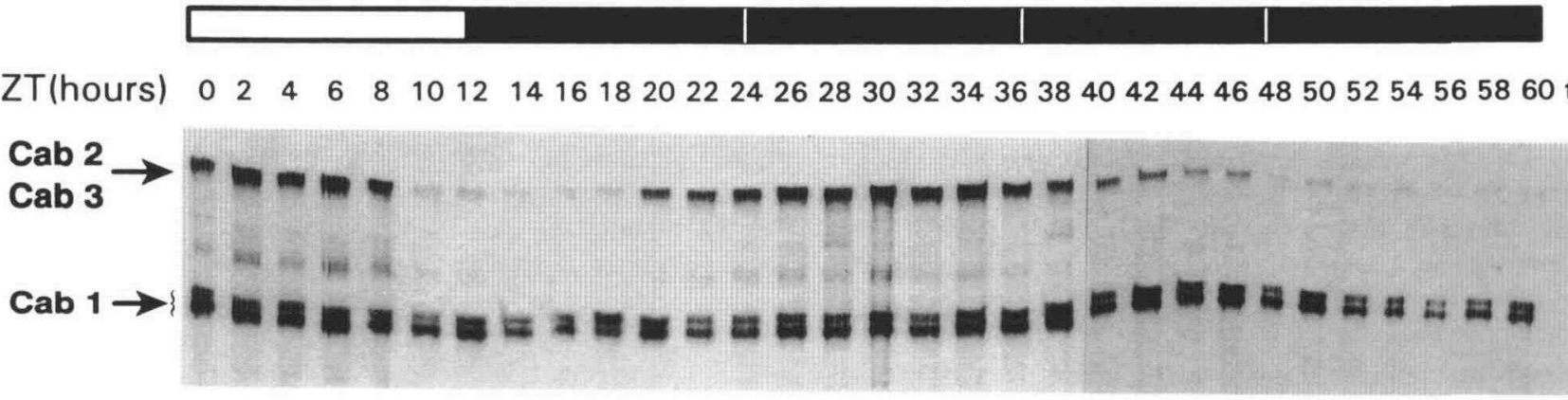

Figure 2. S1 Nuclease Protection Analysis of the cab Family Members in Arabidopsis.

The RNA samples described in Figure 1 were hybridized to a probe from the $5^{\prime}$ end of the cab2 gene. The cab2 and cab3 protection products migrate at the same position (Cab2/Cab3); the cab1 protection products migrate as a double band (Cab1). Open box, light period; filled box, dark period. Each sample contained $25 \mu \mathrm{g}$ of total RNA.

Figure 3 shows the results of a typical experiment from plants bearing either a cab1-cat or cab2-cat translational fusion. The cab1-cat construct exhibited a weak oscillation in the LD cycle, with only a sevenfold difference between maximum (ZTO and ZT27) and the minimum (ZT15) expression levels. In DD, however, we observed a broad plateau in cat mRNA which remained high at least until ZT42. As a control, the level of mRNA encoding the $\beta$-subunit of the endogenous mitochondrial ATPase was assayed on the same blot and was constant throughout the entire experimental period.

In contrast to the cab1-cat expression pattern, the cab2cat translational construct was clearly under circadian regulation, as shown in Figure 3B. The mRNA derived from this construct peaked at ZT3 and at ZT24 and had welldefined peaks in both LD and DD. Expression of this construct declined to a low level after ZT33, and no significant change was observed in the level of the control $\beta$-subunit mRNA throughout this period. All of the independent transformants we assayed gave an expression pattern identical to that shown in Figures $3 \mathrm{~A}$ and $3 \mathrm{~B}$ (data not shown). We also analyzed similar cab3-cat translational fusions that showed expression patterns identical to those of the cab2-cat constructs shown in Figure 3B (data not shown). These data demonstrated that sequences residing between -734 and +462 of the Arabidopsis cab2 gene are sufficient to confer circadian regulation on the cat reporter gene in transgenic tobacco. In the case of the cab1 construct (Figure $3 \mathrm{~A}$ ), only a weak diurnal regulation could be observed, with no detectable cycling in DD.

To investigate further the differential regulation of the cab1 and cab2 genes, we made constructs consisting of transcriptional fusions between the respective cab $5^{\prime}$ upstream regions and the cat reporter gene. No Arabidopsis cab mRNA coding sequence is present in these constructs except for 6 bases of $c a b 1$ and 3 bases of cab2 from the respective $5^{\prime}$ leaders; this allowed us to test whether the clock is acting at the transcriptional level. These constructs were transferred to tobacco, and young Ro plants from several independent transformants were analyzed for circadian activity of the cab promoters, as described above for the translational fusions. Figure $4 \mathrm{~A}$ shows the results of such an analysis for plants containing a construct extending from -249 to +6 of the cab1 promoter fused to cat and sampled at 4-hr intervals. In sharp contrast to the translational cab1 fusion (Figure $3 \mathrm{~A}$ ), the transcriptional fusion showed a clear circadian fluctuation in both LD and DD (Figure 4A). In LD, the first peak in cat mRNA occurred at ZT4 and the second peak in the ZT28 sample in DD, whereas the mRNA level declined to a low level at ZT32. No broad plateau in mRNA level occurred in DD as we observed for the translational fusion (Figure 3A). The amplitude of expression between the maxima (ZT4 and ZT28) and minima (ZT12 and ZT32) was 25-fold, which is much larger than that observed for the translational fusion. Some damping of the rhythm is evident because the peak at ZT28 was only $70 \%$ of the peak at ZT4, as would be expected for circadian regulation. As a control, we analyzed the expression of a cauliflower mosaic virus $35 \mathrm{~S}$ promoter- $\beta$-glucuronidase (gus) reference gene that is also present on the T-DNA, and we found that it does not exhibit circadian-regulated expression (Figure 4A).

We also analyzed the expression of a construct containing the cab2 $5^{\prime}$ upstream region extending from -319 to +3 fused to the cat coding sequence in the same vector used for the cab1-cat transcriptional fusion described above. Transgenic tobacco seedlings from several independent transformants bearing this construct were analyzed by RNA gel blot analysis, and Figure 4B shows a typical example. The expression of this transcriptional cab2-cat chimeric gene was regulated in a circadian fashion in a pattern very similar to that observed for the 
A

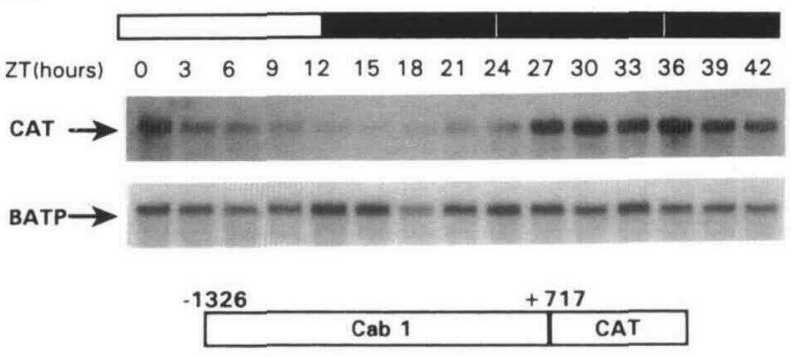

B

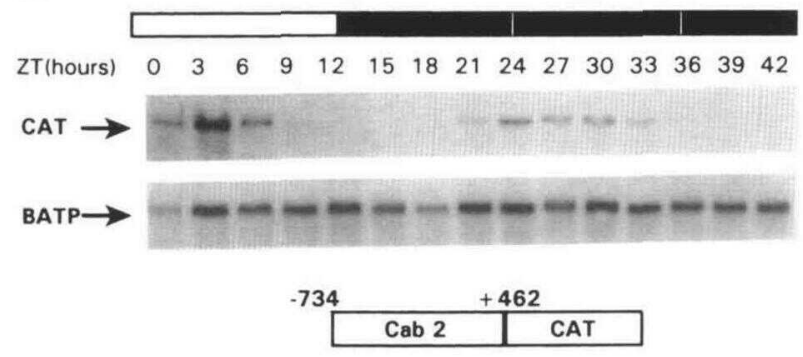

Figure 3. RNA Gel Blot Analysis of cab-cat Translational Fusions in Transgenic Tobacco.

(A) cab1-cat translational fusion.

(B) cab2-cat translational fusion.

Total RNA was prepared from the relevant transgenic plants harvested at the times indicated. An RNA gel blot of these samples was hybridized to coding region probes from cat (CAT) and the $\beta$-subunit of the mitochondrial ATPase (BATP). Open box, light period; filled box, dark period. Boxes below each part: nucleotide positions of $c a b$ fragments used in each construction, and relative length of the cat coding region. Twenty micrograms of total RNA was loaded in each lane.

translational cab2-cat fusion described earlier (Figure 3B) and the transcriptional cab1-cat fusion (Figure 4A). The first peak in the cat mRNA level occurred around ZT3 in LD and the second peak around ZT27 in DD, with the second peak damped by $35 \%$ relative to the first. The peak mRNA level at ZT3 was 50 -fold greater than the trough at ZT15.

To confirm that the transcription rate of the cab1 and cab2 genes is circadian regulated in Arabidopsis, we performed in vitro nuclear run-on transcription assays. Nuclei were prepared from Arabidopsis plants through one cycle of LD, followed by one cycle of DD. The nuclei were used to generate nascent radiolabeled transcripts in vitro, which were then hybridized to filters bearing single-strand DNA probes derived from either the cab1 or cab2 genes, or rDNA as a control. Figure 5 shows the results of a typical experiment, where transcription rate is represented as the fraction of total input ${ }^{32} \mathrm{P}$-RNA that hybridized to each probe. Both the cab1 and cab2 genes exhibited a transcription rate that dropped markedly from ZT4 to a trough at ZT10 to ZT16 and peaked again at ZT28 in DD, indicating circadian regulation (Figure $5 \mathrm{~A}$ ). We note that the timing of peaks and troughs appears to precede by several hours the corresponding maxima and minima in mRNA levels of the cab2 gene assayed under a similar regime (Figure 2). However, the sampling interval of the nuclei is $6 \mathrm{hr}$, and more frequent time points may be necessary to resolve more accurately the circadian fluctuations in transcription rate. Because of the different probes used for the cab1 and cab2 genes and a low level of cross-hybridization (see Methods), no conclusions can be drawn regarding the relative magnitude of transcription rate between these two genes. In marked contrast to the cab genes, the transcription rate of the rDNA genes appears to exhibit an erratic fluctuation of no more than 1.5-fold throughout the experimental period (Figure 5B). This is very consistent with fluctuations observed for rDNA transcription rate in nuclei isolated from oat seedlings (Lissemore and Quail, 1988).

A

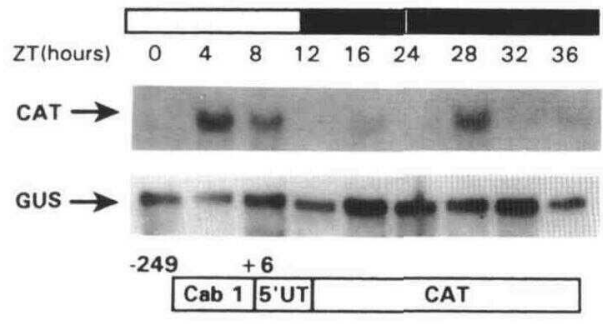

B

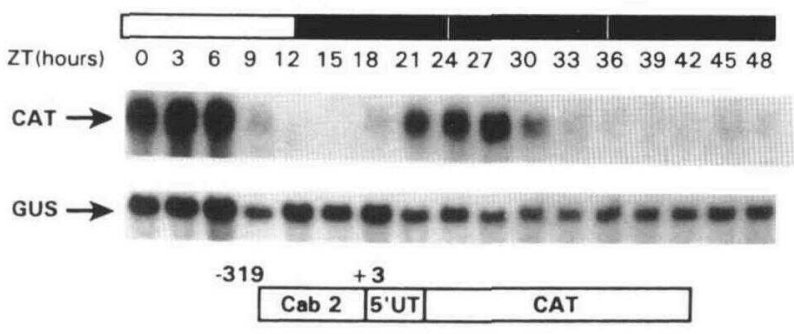

Figure 4. RNA Gel Blot Analysis of cab-cat Transcriptional Fusions in Transgenic Tobacco.

(A) cab1-cat transcriptional fusion.

(B) cab2-cat transcriptional fusion.

Total RNA prepared from plants harvested at the times indicated was hybridized to coding region probes from cat (CAT) and gus (GUS). Open box, light period; filled box, dark period. Boxes below each part: nucleotide positions of cab fragments used in each construction, and relative length of the cat coding region. $5^{\prime} \mathrm{UT}$, $5^{\prime}$ untranslated leader. Twenty micrograms of total RNA was loaded in each lane. 

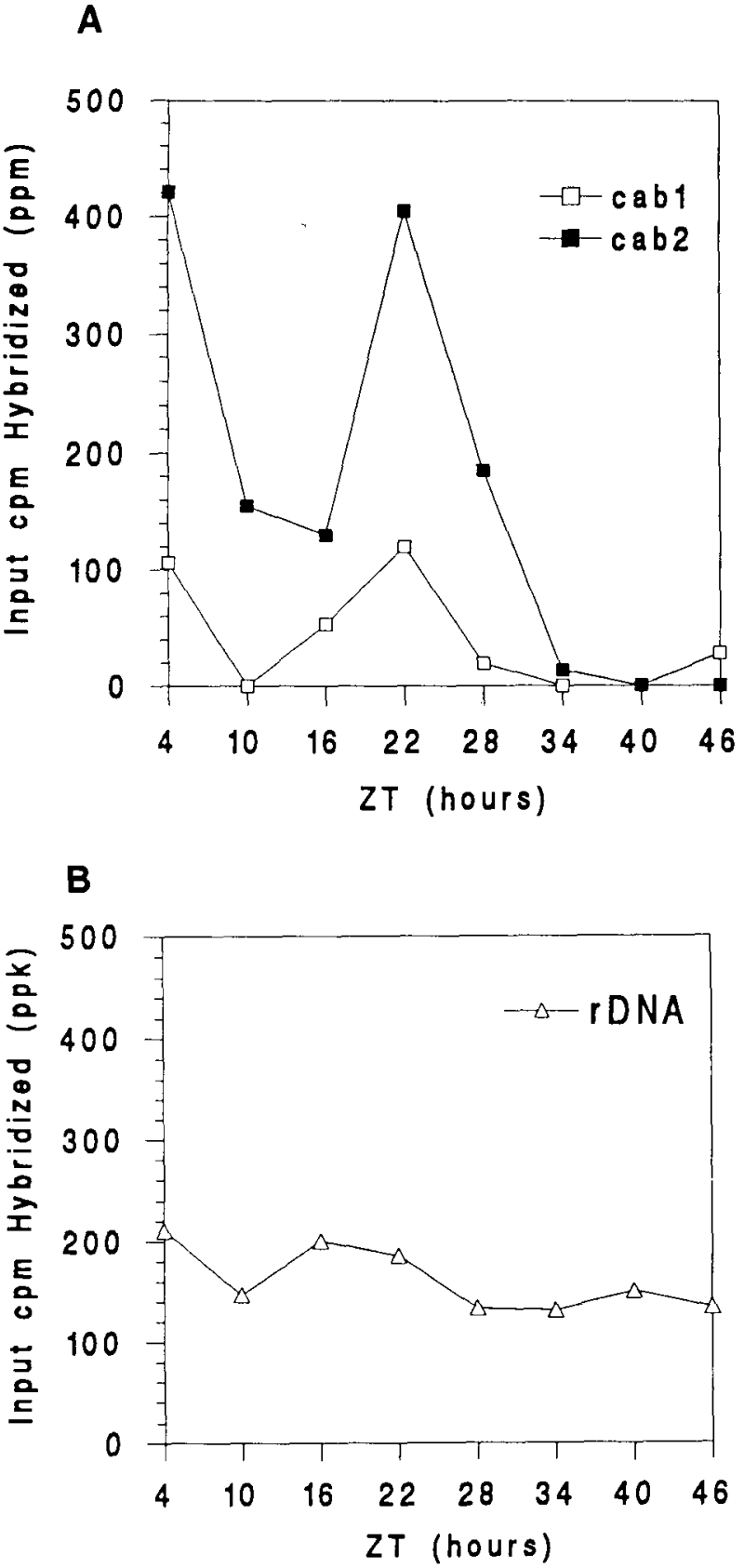

Figure 5. In Vitro Nuclear Run-On Assays of cab Gene Transcription in Arabidopsis.

(A) cab1 and cab2 transcription assays.

(B) rDNA transcription assays.

In vitro transcripts from nuclei prepared at the times indicated were hybridized to probes derived from the cab1 and cab2 $3^{\prime}$ untranslated regions $(\square, \square)$ and to rDNA $(\triangle)$. Lights were on from ZTO to ZT8 during plant harvesting, followed by constant darkness to ZT46. Data for rRNA hybridization is presented as parts per thousand (ppk).
Taken together, all of these data demonstrated that transcription of both the Arabidopsis cab1 and cab2 genes is regulated by the circadian clock and occurs in a similar phase. The transcriptional regulation is mediated by cisacting elements residing between -249 and +6 in cab1 and -319 and +3 in cab2. Furthermore, a post-transcriptional mechanism may be involved in determining steadystate cab1 mRNA levels in Arabidopsis because the mRNA level does not oscillate significantly but remains relatively constant throughout the diurnal cycle.

\section{DISCUSSION}

The study of circadian clocks has been facilitated in recent years by the concerted application of both genetic and molecular techniques. Genetic loci that are required for normal clock functions have been identified and cloned in Drosophila (Rosbash and Hall, 1989; Young et al., 1989) and Neurospora (Dunlap, 1990). Such loci provide powerful tools for the study of the cellular components of biological clocks. In higher plants, genes required for clock function have not yet been identified, but the discovery of clockregulated mRNAs and cis-acting elements (Nagy et al., 1988a) provides a molecular assay for the circadian clock. We have demonstrated here that clock function can be assayed using relatively small promoter fragments derived from members of the Arabidopsis cab gene family. This will allow the consolidation of molecular and genetic approaches to study clocks in a model system for higher plants. Interestingly, we have also demonstrated that the steady-state level of cab1 mRNA does not cycle in Arabidopsis, whereas the cab1 promoter confers circadianregulated expression in transgenic tobacco. This result provides a clear example of the importance of post-transcriptional events in determining steady-state mRNA levels of specific plant genes, which is currently an area of intense interest (Walling et al., 1986; Dean et al., 1989; Ingelbrecht et al., 1989; Silverthorne and Tobin, 1990).

Our results showed that the cab1 upstream region confers high amplitude cycling on the cat reporter gene in transcriptional fusions (Figure 4A), whereas translational cab1-cat fusions exhibit a low amplitude rhythm in LD (Figure 3A) and the endogenous steady-state cab1 mRNA level exhibits a very weak oscillation, if any. Several factors must be considered that may account for these data. First, the upstream regions present in the translational and transcriptional fusions are different, and this may be the only reason for the observed differences in reporter gene expression. The cab1-cat translational fusion contains 1326 bp of $5^{\prime}$ upstream region, whereas the cab1-cat transcriptional fusion only contains $249 \mathrm{bp}$ upstream of the transcriptional initiation site (Figures $3 \mathrm{~A}$ and $4 \mathrm{~A}$ ). Sequences present in the additional $1 \mathrm{~kb}$ of upstream region, 
therefore, may have given rise to the relatively low amplitude cycling observed with the translational fused construct. We consider this explanation unlikely because we have also assayed the expression of a cab1-cat translational fusion (provided by Dr. An) containing only 251 bp of upstream sequence and this construct exhibited an expression pattern of low amplitude cycling identical to that observed for the -1326 fusion (A. J. Millar and S. A. Kay, unpublished results). Second, the vector used for the $c a b$-cat translational constructs is different from that used for our transcriptional fusions, creating different $3^{\prime}$ untranslated regions in the chimeric mRNAs that may have also influenced our results. However, the cab2-cat fusions gave an identical expression pattern regardless of vector background (Figures $3 \mathrm{~B}$ and $4 \mathrm{~B}$ ), indicating that this did not have a significant effect on our data.

Another possible explanation for the discrepancy between the cab1 promoter expression pattern and the steady-state cab1 mRNA level in Arabidopsis is that the promoter constructs were all assayed in transgenic tobacco rather than in Arabidopsis. Thus, the cab1 promoter could be regulated inappropriately in tobacco. To investigate this, we performed in vitro nuclear run-on experiments in Arabidopsis to test for cab transcriptional activity throughout a circadian cycle in DD and found that the transcription of both the cab1 and cab2 genes cycles throughout this period (Figure 5). Therefore, it is highly likely that the cab1 promoter is circadian responsive in both Arabidopsis and transgenic tobacco. In further support of this conclusion, previous studies have shown that all three Arabidopsis cab gene promoters exhibit a pattern of expression in transgenic tobacco very similar to that observed in Arabidopsis ( $\mathrm{Ha}$ and An, 1988; Mitra et al., 1989).

The most plausible hypothesis that accounts for our data is that sequences downstream of +6 in the cab1 gene are required for a post-transcriptional mechanism that results in cab1 mRNA levels remaining relatively constant throughout the diurnal cycle. This occurs despite the clock-regulated transcription of the cab1 gene. Interestingly, the sequences mostly responsible for this effect may reside in the $5^{\prime}$ untranslated leader of the cab1 gene. Support for this notion comes from analysis of the translational cab-cat fusions. The transcribed cab coding sequences present in these constructs extend from the initiation codon at +71 to +717 in the case of cab1 and from the initiation ATG at +54 to +462 for the cab2 construct. The $c a b 1$ and $c a b 2$ coding sequences from the ATG to +717 are $96 \%$ homologous with only single-base pair mismatches (Leutweiler et al., 1986). Thus, the difference in expression pattern of cab1-cat versus cab2-cat translational fusions is unlikely to be due to the Cab protein coding sequences retained in these constructs.

A more plausible candidate responsible for the differential expression pattern is the $5^{\prime}$ untranslated leader of $c a b 1$, which is divergent from the cab2 and cab3 leader sequences. If this hypothesis were true, it would be one of the first examples in which the $5^{\prime}$ untranslated mRNA leader has been implicated in regulating mRNA levels in higher plants. Future experiments placing the cab1 leader in cab2-cat fusions will determine whether the cab1 $5^{\prime}$ leader has an independent role in post-transcriptional regulation. We note that the cab1-cat translational fusion exhibits a higher amplitude circadian expression pattern than the steady-state cab1 mRNA level observed in Arabidopsis. This difference could be because of two hypothetical mechanisms. First, the translational cab1-cat construct (Figure 3A) may require additional sequences from the 3 ' portion of the cab1 gene to generate the expression pattern observed in Arabidopsis (Figure 2). Second, the presence of the cat mRNA coding sequences, which we have shown previously to be relatively unstable in transgenic tobacco (Nagy et al., 1988a), may give rise to some of the cycling seen in the cab1-cat chimeric RNA. We cannot yet account for the observation that the translational cab1-cat fusion does not cycle at all in constant darkness (Figure $3 \mathrm{~A}$ ) but shows a low amplitude cycling in LD. Further experiments will determine the expression pattern of the chimeric cab1-cat mRNA in extended darkness.

The physiological consequences of circadian-regulated cab gene expression in Arabidopsis are currently unknown. We have proposed previously (Nagy et al., 1988a; Kay and Millar, 1990) that cycling of cab mRNA may be a mechanism by which synthesis of $c a b$ apoprotein could be coupled to light-regulated chlorophyll biosynthesis. This could be important in both the biogenesis and maintenance of light-harvesting complexes in vivo. However, it is unclear why the cab1 gene is clock regulated at the transcriptional level when this control does not manifest itself at the level of steady-state cab1 mRNA. Our data have demonstrated that the closely related cab2 and cab3 genes are cycling at the transcriptional level as well as at the level of mRNA. All three cab genes encode identical mature proteins, which we presume are functionally equivalent, but it is not known whether all three genes are expressed in the same cell types. Previous experiments showed that the cab1 gene is induced by light to a greater extent than the cab2/ 3 in etiolated tissue (Karlin-Neumann et al., 1988). However, it has not been demonstrated whether the differential regulation is exerted at the level of transcription or by posttranscriptional events. This expression pattern may have evolved to deliver the cab1 and cab2/3 gene products to distinct groups of cells at appropriate times. We are currently investigating the cell-specific expression pattern of the individual Arabidopsis cab genes using upstream regions fused to luciferase (Koncz et al., 1990) reporter genes.

The upstream cis-elements identified in the wheat cab1 gene (Nagy et al., 1988a; Fejes et al., 1990) and the three Arabidopsis cab genes reported here are the only such elements that are known to confer circadian-regulated 
transcription. per mRNA has been shown to cycle in Drosophila (Hardin et al., 1990), and clock-controlled genes (ccgs) that are transcriptionally regulated have been identified in Neurospora (Loros and Dunlap, 1991), but no upstream elements conferring this regulation have yet been identified. The cab circadian-responsive cis-elements that we have identified in Arabidopsis will allow two fundamental approaches for dissecting the circadian clock. The first is a molecular approach that will allow a more detailed definition of the clock-responsive elements, which in turn can be used to assay for putative protein factors whose activity is regulated in a time-dependent manner. Such proteins are already known to exist, as the level of a mammalian transcription factor, DBP, was shown to be circadian regulated in rat hepatocytes (Wuarin and Schibler, 1990). The second approach is a genetic analysis in which the cab upstream region, fused to either screenable markers [such as luciferases lux (Koncz et al., 1987) and luc (Schneider et al., 1990) or gus (Jefferson et al., 1987)], or selectable markers, such as hygromycin phosphotransferase, will allow identification of loci that are responsible for correct clock function in Arabidopsis.

\section{METHODS}

\section{Plant Materia}

Arabidopsis thaliana plants (ecotype Columbia g/1, seed supplied by Guhy's Specialty Nursery, Tucson, AZ) were grown in a growth chamber at a constant $22^{\circ} \mathrm{C}$ under a 12-hr photoperiod (12L12D) for 3 weeks (RNA analysis) or under 8L16D for 5 weeks (nuclear isolation). R1 seeds of transformed tobacco (var Xanthi) carrying cab-cat translational fusions $(A n, 1987)$ were grown in sterile culture on MS medium (Murashige and Skoog, 1962) containing kanamycin at $23^{\circ} \mathrm{C}$ for 2 weeks under $16 \mathrm{~L} 8 \mathrm{D}$, then for 2 days in 12L12D. Tobacco plants (var SR1) were maintained in sterile culture on MS medium to provide leaves for transformation experiments. Regenerated kanamycin-resistant plants carrying the transcriptional cab-cat fusions were propagated in sterile culture and entrained to $12 \mathrm{~L} 12 \mathrm{D}$ as for the R1 plants. Three to five independently transformed lines that expressed the transgene at high levels were assayed for each of the transcriptional and translational fusions.

\section{RNA Extraction and Analysis}

Total RNA was prepared from all aerial parts of the plants (Arabidopsis and translational cab-cat fusions) or from leaf tissue (transcriptional fusions) and analyzed in slot blots, RNA gels, or S1 nuclease protection assays, as described previously (Nagy et al., 1988b). The coding region probes used in the slot blots and RNA gel blots were from the $\beta$-subunit of the mitochondrial ATPase (Boutry and Chua, 1985) and a 537-bp Avall fragment from cab1. The probe used in Figure 2 was a $640-b p$ Clal-BamHI fragment from the $5^{\prime}$ end of the cab2 gene, uniformly labeled with ${ }^{35} \mathrm{~S}$-deoxyadenosine. Hybridization for the $\mathrm{S} 1$ nuclease protection assays was performed with $25 \mu \mathrm{g}$ of total RNA in $80 \%$ formamide/ $0.4 \mathrm{M} \mathrm{NaCl}$ at $47^{\circ} \mathrm{C}$, with digestion in 800 units $/ \mathrm{mL} \mathrm{S} 1$ nuclease for $90 \mathrm{~min}$ at room temperature. The double band produced by the cab1 mRNA protection product (about 155 nucleotides) is a property specific to this probe; the cab2 and cab3 protection products (206 nucleotides) comigrate. Autoradiograms of the slot blots, RNA gel blots, and S1 analysis were quantitated by densitometry using an LKB Ultroscan densitometer. The amplitude of $c a b$ or cat mRNA cycling in the slot blots and RNA gel blots was calculated from the ratios of cab or cat to control ( $\beta$-ATPase or gus) density for the relevant time points. The amplitude of mRNA cycling in the $\mathrm{S} 1$ analysis was calculated from the absolute density of the relevant bands.

\section{Clones and Construction}

Arabidopsis genomic clones containing the cab1 (AB140) and cab2 (AB165) genes were kindly provided by Dr. Elaine Tobin (University of California, Los Angeles). All nucleotide positions are calculated using the transcription start sites defined previously (Karlin-Neumann et al., 1988) as the +1 position. The R1 tobacco seeds kindly provided by Dr. Gynheung An carry cat translational fusions $568(-1326$ to +717 of cab1), $499(-734$ to +462 of $c a b 2)$, and 500 ( -900 to +462 of cab3). Briefly, the translational fusions consist of the relevant cab-cat fusion described above in the binary cat expression vector PGA534 and have been described in detail (An, 1987). All the translational fusions contain the same cat sequences and octopine T-DNA $3^{\prime}$ termination signal. The transcriptional fusions to cat contain the following sequences: in cab1-cat, a Bglll-SfaNl fragment (-249 to -3) of cab1 is fused to an oligonucleotide that reconstitutes the cab1 sequence from -249 to +6 , followed by a BspMI site, 29 bp of the wheat cab1 $5^{\prime}$ untranslated region, and a Sall site at position +47 of the fusion construct. The heterologous $5^{\prime}$ untranslated region was included to eliminate possible post-transcriptional effects of Arabidopsis mRNA sequences on MRNA abundance. Nagy et al. (1988a) demonstrated that the abundance of the wheat cab1 transcript does not cycle in transgenic tobacco when fused to a constitutive promoter. The cab2-cat transcriptional fusion contains a Taql fragment of cab2 $(-319$ to -16$)$ fused to an oligonucleotide that reconstitutes cab2 sequence from -319 to +3 , followed by sequences identical to the cab1-cat construct from +4 to the Sall site. These promoter-leader constructs were ligated to an identical cat gene bearing the pea rbcS-E9 transcriptional terminator in the pUC-based plasmid $\mathrm{p} 827$, provided by Dr. Pam Green (Michigan State University), and each cab-cat-E9 fusion was transferred to the polylinker of the binary vector pMON721 (provided by Monsanto Corporation, St. Louis, MO). A reference gene consisting of the cauliflower mosaic virus $35 \mathrm{~S}$ promoter from position -941 , fused to the gus gene with the pea rbcS-3C transcriptional terminator, was introduced into the Notl site at position 4836 of pMON721, reading on the opposite strand from the cab-cat fusion.

\section{In Vitro Nuclear Run-On Transcription Assays}

Nuclei were isolated at 6-hr intervals from all aerial parts of prefloral Arabidopsis plants as described (Green et al., 1988). The time to prepare nuclei, from tissue homogenization to freezing, 
was approximately $30 \mathrm{~min}$. In vitro nuclear run-on assays were performed essentially as described (Lissemore and Quail, 1988). The cab $3^{\prime}$ untranslated region probes were isolated by polymerase chain reaction from genomic DNA and several independent clones were sequenced for each construct. The cab1 probe corresponds to nucleotides +767 to +866 . The cab2 probe spanned the 1.6-kb region between cab2 and cab3, from position +769 of cab2 to position -878 of cab3. The Arabidopsis rDNA clone pARR17 was kindly provided by Dr. Eric Richards (Cold Spring Harbor). Single-stranded DNA probes and ${ }^{35} \mathrm{~S}$ in vitro transcripts were prepared from clones in pBluescript vectors, as recommended by the manufacturer (Stratagene). Single-stranded DNA probes were immobilized on a nitrocellulose filter using a slot-blot manifold. Filters were cut and individual slots hybridized in 1.5-mL microcentrifuge tubes. Hybridization and washing conditions were as described (Lissemore and Quail, 1988) except that the final wash was at $37^{\circ} \mathrm{C}$ in $1 \times \mathrm{SSC} / 0.5 \%$ SDS. cab and rRNA hybridizations were normalized by subtracting ${ }^{32} \mathrm{P}$ hybridization to pBluescript as a background control. Controls were also performed using ${ }^{35}$ S-RNAs to test for cross-hybridization between the single-stranded DNA probes and RNA from the noncognate cab gene. Hybridization of input probe counts per minute after background subtraction were 13\% for the cab2 RNA on the cab1 probe and $28 \%$ for the cab1 RNA on the cab2 probe. Hybridization efficiency of $c a b$ and rDNA transcripts was calculated by incorporating a known amount of ${ }^{35} \mathrm{~S}$ RNA in each of the hybridization reactions. The assays were repeated six times and gave consistent results. Figure 5 shows the results of typical experiments, in which hybridization efficiencies ranged from $26 \%$ to $40 \%$ for cab probes and $42 \%$ to $74 \%$ for the rDNA probes. The cab and rRNA data in this figure are derived from separate assays of the same nuclear samples.

\section{ACKNOWLEDGMENTS}

We wish to thank Irene Roberson, Ellen Leheny, and Stanley Sotnikov for expert technical assistance. We are also grateful to Dr. Elaine Tobin for providing the Arabidopsis cab1 and cab2 genes, to Dr. Gynheung An for the generous gift of transgenic tobacco seeds containing cab-cat translational fusions, and to Dr. Alan Aderem for the use of an LKB Ultroscan densitometer. This work was supported by a Rockefeller Foundation grant to NamHai Chua (Rockefeller University).

Received February 21, 1991; accepted March 19, 1991.

\section{REFERENCES}

An, G. (1987). Integrated regulation of the photosynthetic gene family from Arabidopsis thaliana in transformed tobacco cells. Mol. Gen. Genet. 207, 210-216.

Boutry, M., and Chua, N.-H. (1985). A nuclear gene encoding the $\beta$ subunit of the mitochondrial ATPase in $N$. plumbaginifolia. EMBO J. 4, 2159-2165.
Dean, C., Favreau, M., Bond-Nutter, D., Bedbrook, J., and Dunsmuir, P. (1989). Sequences downstream of translation start regulate quantitative expression of two petunia $r b c S$ genes. Plant Cell 1, 201-208.

Deng, M.-D., Moureaux, T., and Lamaze, T. (1989). Diurnal and circadian fluctuation of malate levels and its close relationship to nitrate reductase activity in tobacco leaves. Plant Sci. $\mathbf{6 5}$, 191-197.

Dunlap, J.C. (1990). Closely watched clocks: Molecular analysis of circadian rhythms in Neurospora and Drosophila. Trends Genet. 6, 159-165.

Edmunds, L.N. (1988). Cellular and Molecular Bases of Biological Clocks. (New York: Springer-Verlag).

Estelle, M.A., and Somerville, C. (1986). The mutants of Arabidopsis. Trends Genet. 2, 89-93.

Fejes, E., Pay, A., Kanevsky, I., Szell, M., Adam, E., Kay, S., and Nagy, F. (1990). A 268 bp upstream sequence mediates the circadian clock-regulated transcription of the wheat cab-1 gene in transgenic plants. Plant Mol. Biol. 15, 921-932.

Giuliano, G., Moffman, N.E., Ko, K., Scolnik, P.A., and Cashmore, A.R. (1988). A light-entrained circadian clock controls transcription of several plant genes. EMBO J. 7, 3635-3642.

Gorton, H.L., Williams, W.E., Binns, M.E., Gemmell, C.N., Leheny, E.A., and Shepherd, A.C. (1989). Circadian stomatal rhythms in epidermal peels from Vicia faba. Plant Physiol. 90, 1329-1334.

Green, P.J., Kay, S.A., Lam, E., and Chua, N.-H. (1988). In vitro DNA footprinting. In Plant Molecular Biology Manual, S. Gelvin and R. Schilperoort, eds (Dordrecht, The Netherlands: Kluwer Academic Publishers), pp. 1-22.

Ha, S.-B., and An, G. (1988). Identification of upstream regulatory elements involved in the developmental expression of the Arabidopsis thaliana cab1 gene. Proc. Natl. Acad. Sci. USA 85, 8017-8021.

Hardin, P.E., Hall, J.C., and Rosbash, M. (1990). Feedback of the Drosophila period gene product on circadian cycling of its messenger RNA levels. Nature 343, 536-540.

Huang, T.C., Tu, J., Chow, T.-J., and Chen, T.-H. (1990). Circadian rhythm of the prokaryote Synechococcus sp. RF-1. Plant Physiol. 92, 531-533.

Ingelbrecht, I.L.W., Herman, L.M.F., Dekeyser, R.A., Van Montagu, M.C., and Depicker, A.G. (1989). Different $3^{\prime}$ end regions strongly influence the level of gene expression in plant cells. Plant Cell 1, 671-680.

Jefferson, R.A., Kavanagh, T.A., and Bevan, M.W. (1987). GUS fusions: $\beta$-Glucuronidase as a sensitive and versatile gene fusion marker in higher plants. EMBO J. 6, 3901-3907.

Karlin-Neumann, G.A., Sun, L., and Tobin, E.M. (1988). Expression of light-harvesting chlorophyll $a / b$-protein genes is phytochrome-regulated in etiolated Arabidopsis thaliana seedlings. Plant Physiol. 88, 1323-1331.

Kay, S. A., and Millar, A. J. (1990). Circadian regulated cab gene transcription in higher plants. In The Molecular Biology of Circadian Rhythms, M. Young, ed (New York: Marcel Dekker), in press. 
Koncz, C., Olsson, O., Langridge, W.H.R., Schell, J., and Szalay, A.A. (1987). Expression and assembly of functional bacterial luciferase in plants. Proc. Natl. Acad. Sci. USA 84, 131-135.

Koncz, C., Langridge, W.H.R., Olsson, O., Schell, J., and Szalay, A.A. (1990). Bacterial and firefly luciferase genes in transgenic plants: Advantages and disadvantages of a reporter gene. Dev. Genet. 11, 224-232.

Lecharny, A., and Wagner, E. (1984). Stem elongation rate in light-grown plants: Evidence for an endogenous circadian rhythm in Chenopodium rubrum. Physiol. Plant. 60, 437-453.

Lecharny, A:; Tremolières, A., and Wagner, E. (1990). Correlation between the endogenous circadian rhythmicity in growth rate and fluctuations in oleic acid content in expanding stems of Chenopodium rubrum L. Planta 182, 211-215.

Leutweiler, L.S., Meyerowitz, E.M., and Tobin, E.M. (1986). Structure and expression of three light-harvesting chlorophyll $a / b$-binding protein genes in Arabidopsis thaliana. Nucl. Acids Res. 14, 4051-4064.

Lissemore, J.L., and Quail, P.H. (1988). Rapid transcriptional regulation of the genes for phytochrome and chlorophyll $a / b$ binding protein in Avena sativa. Mol. Cell. Biol. 8, 4840-4850.

Loros, J.J., and Dunlap, J.C. (1991). Neurospora crassa clockcontrolled genes are regulated at the level of transcription. Mol. Cell. Biol. 11, 558-563.

Meyer, H., Thienel, U., and Piechulla, B. (1989). Molecular characterization of the diurnal/circadian expression of the chlorophyll $a / b$-binding proteins in leaves of tomato and other dicotyledonous and monocotyledonous plant species. Planta 180, 5-15.

Millet, B., Coillot, L., and Agosti, R.D. (1989). The rhythmic leaf movements after regeneration of partially excised pulvinus in Phaseolus vulgaris L. Plant Cell Physiol. 30, 643-648.

Mitra, A., Choi, H.K., and An, G. (1989). Structural and functional analyses of Arabidopsis thaliana chlorophyll $a / b$-binding protein (cab) promoters. Plant Mol. Biol. 12, 169-179.

Murashige, T., and Skoog, F. (1962). A revised medium for rapid growth and bioassays with tobacco tissue culture. Physiol. Plant. 15, 493-497.

Nagy, F., Kay, S.A., and Chua, N.-H. (1988a). A circadian clock regulates transcription of the wheat cab-1 gene. Genes Dev. 2, 376-382.

Nagy, F., Kay, S.A., and Chua, N.-H. (1988b). Analysis of gene expression in transgenic plants. In Plant Molecular Biology
Manual, S. Gelvin and R. Schilperoort, eds (Dordrecht, The Netherlands: Kluwer Academic Publishers), pp. 1-29.

Paulsen, H., and Bogorad, L. (1988). Diurnal and circadian rhythms in the accumulation and synthesis of mRNA for the light-harvesting chlorophyll $a / b$-binding protein in tobacco. Plant Physiol. 88, 1104-1109.

Redei, G.P. (1970). Arabidopsis thaliana (L.) Heynh: A review of the genetics and biology. Bibliogr. Genet. 20, 1-151.

Rosbash, M., and Hall, J.C. (1989). The molecular biology of circadian rhythms. Neuron 3, 387-398.

Schneider, M., Ow, D.W., and Howell, S.H. (1990). The in vivo pattern of firefly luciferase expression in transgenic plants. Plant Mol. Biol. 14, 935-947.

Silverthorne, J., and Tobin, E.M. (1990). Post-transcriptional regulation of organ-specific expression of individual $r b c S$ mRNAs in Lemna gibba. Plant Cell 2, 1181-1190.

Simon, E., Satter, R.L., and Galston, A.W. (1976). Circadian rhythmicity in excised Samanea pulvini. II. Resetting the clock by phytochrome conversion. Plant Physiol. 58, 421-425.

Stayton, M.M., Brosio, P., and Dunsmuir, P. (1989). Photosynthetic genes of Petunia (Mitchell) are differentially expressed during the diurnal cycle. Plant Physiol. 89, 776-782.

Sweeney, B.M. (1987). Rhythmic Phenomena in Plants. (San Diego: Academic Press).

Taylor, W.C. (1989). Transcriptional regulation by a circadian rhythm. Plant Cell 1, 259-264.

Uhl, G.R., and Reppert, S.M. (1986). Suprachiasmatic nucleus vasopressin messenger RNA: Circadian variation in normal and Brattleboro rats. Science 232, 390-393.

Walling, L., Drews, G.N., and Goldberg, R.B. (1986). Transcriptional and post-transcriptional regulation of soybean seed protein mRNA levels. Proc. Natl. Acad. Sci. USA 83, 2123-2127.

Wuarin, J., and Schibler, U. (1990). Expression of the liverenriched transcriptional activator protein DBP follows a stringent circadian rhythm. Cell 63, 1257-1266.

Young, M.W., Bargiello, T.A., Baylies, M.K., Saez, L., and Spray, D.C. (1989). Molecular biology of the Drosophila clock. In Neuronal and Cellular Oscillators, J.W. Jacklet, ed (New York: Marcel Dekker), pp. 529-542.

Zerr, D.M., Hall, J.C., Rosbash, M., and Siwicki, K.K. (1990). Circadian fluctuations of period protein immunoreactivity in the CNS and the visual system of Drosophila. J. Neurosci. 10, 2749-2762. 